\title{
Spatiotemporal Frequency and Direction Sensitivities of Human Visual Areas Measured Using fMRI
}

\author{
K. D. Singh, ${ }^{* 1}$ A. T. Smith,* and M. W. Greenleet ${ }^{, 2}$ \\ *Royal Holloway College, University of London, Egham, England; and †Neurologische und \\ Radiologische Universitätskliniken, Freiburg, Germany
}

Received December 28, 1999

\begin{abstract}
Using functional magnetic resonance imaging (fMRI) we have studied the variation in response magnitude, in each visual area (V1-V5), as a function of spatial frequency (SF), temporal frequency (TF) and unidirectional motion versus counterphase flicker. E ach visual area was identified in each subject using a combination of retinotopic mapping $\mathrm{fMRI}$ and cortical flattening techniques. A drifting (or counterphasing) sinusoidal grating was used as the stimulus in a study in which we parametrically varied SF between 0.4 and 7 cycles/degree and TF between 0 and $18 \mathrm{~Hz}$. For each experiment we constructed fMRI amplitude tuning curves, averaged across subjects, for each visual area. The tuning curves that resulted are consistent with the known physiological properties of cells in the corresponding macaque visual areas, previous functional imaging studies, and in the case of V1, the psychophysically determined contrast sensitivity functions for spatial and temporal frequency. In the case of V3A, the SF tuning functions obtained were more similar to those found in single cell studies of macaque V 3 rather than macaque V3A. All areas showed at least a moderate preference for directed versus counterphasing motion with V5 showing the largest preference. Visual areas V1, V2, V3, and V3A showed more direction sensitivity at low spatial frequencies, while VP, V4, and V5 had the highest drifting versus counterphasing ratios for higher spatial frequencies.
\end{abstract}

\section{INTRODUCTION}

Primate and human visual systems consist of anatomically discrete areas, many of which contain retinotopically organized maps of visual space. The functional specificity of these areas was initially formulated in terms of the response properties of the neurons

\footnotetext{
${ }^{1}$ Present address: MARIARC, Liverpool University, Liverpool, England.

${ }^{2}$ Present address: Department of Psychology, University of Oldenburg, Oldenburg, Germany.
}

within each area, using single-cell recordings in animals. For example, neurons within an area known as V5 or MT (Allman and Kaas, 1971; Dubner and Zeki, 1971; Zeki, 1974) show a preference for features which are moving or changing with time (Maunsell and Van Essen, 1983). The spatiotemporal frequency sensitivities of neurons within each visual area are considered fundamental attributes and are often studied. For example, primate studies suggest that V5/MT receives most of its input from the magnocellular pathway (Maunsell et al., 1990), making it optimally sensitive to low spatial frequencies.

Recently developed functional imaging techniques measure macroscopic quantities such as evoked electrical currents, or local hemodynamic changes. Human homologues of the primate visual areas and the retinotopic organization of the human visual system have been described using Positron Emission Tomography (PET) (Fox et al., 1987; Zeki et al., 1991; Watson et al., 1993), Functional Magnetic Resonance I maging (fMRI) (Tootell et al., 1995; Engel et al., 1994, 1997; Sereno et al., 1995) and Magnetoencephalography (MEG) (Anderson et al., 1996; Fylan et al., 1997). In V5/MT it has been demonstrated that the properties of its neuronal population have a direct influence on macroscopic functional imaging signals. Tootell et al. (1995) showed that the magnitude of the hemodynamic response saturates at a low image contrast (5\%) as predicted by the magnocellular predominance in V5/MT. These results were confirmed using MEG (Anderson et al., 1996) who also confirmed the expected low spatial frequency sensitivity of V5/MT.

Spatiotemporal frequency and direction sensitivities of the human visual system can also be studied using psychophysics (e.g., Campbell and Robson, 1968; Kulikowski and Tolhurst, 1973; Kelly, 1979). There is some debate as to whether psychophysical decisions are the result of small (Britten et al., 1992) or large (Shadlen et al., 1996) numbers of neurons firing synchronously. Boynton et al. (1999) showed that psychophysical contrast thresholds were well correlated with increasing fMRI activation. This suggests that behav- 
ioral performance depends on the simultaneous firing of large numbers of neurons, and we would therefore expect a correlation between macroscopic functional imaging measures such as MEG and fMRI, and psychophysical measures.

Although spatial frequency, temporal frequency, and direction sensitivity are considered fundamental attributes of primate and human visual systems, there has not yet been a systematic functional imaging study of how the activation in each visual area varies as a function of these stimulus properties, and we have therefore carried out such an investigation using fMRI . If the hemodynamic response is strongly linked to the underlying neuronal population and psychophysical performance is mediated by the synchronous activity of large numbers of neurons, our results should accord with both primate cell electrophysiology and psychophysical studies in human. Thus, macroscopic functional imaging techniques such as $\mathrm{fMRI}$ could bridge an important gap between these two domains.

\section{MATERIALS AND METHODS}

\section{Subjects}

The subjects were 10 healthy human volunteers (5 male, 5 female), some of whom were paid for their time. All subjects either had normal vision or were corrected for myopia using contact lenses. I nformed consent was obtained in writing.

\section{MRI Data Acquisition}

Imaging was performed using a 1.5T Siemens Magnetom (Vision) scanner, which has $25 \mathrm{mT} / \mathrm{m}$ gradients with a 0.3-ms rise time. The subject was positioned in the $\mathrm{RF}$ receive-transmit full head coil, and the head was stabilized using a vacuum cap.

Functional images were twelve contiguous T2* weighted EPI slices (TE $=66 \mathrm{~ms}$, TR $=3 \mathrm{~s}$, Flip Angle $=90^{\circ}, 128 \times 128$ matrix, voxel size $=2 \times 2 \times 4$ $\mathrm{mm})$, positioned approximately parallel to the calcarine sulcus, and encompassing occipital and posterior parietal lobes.

\section{Visual Stimulation}

All stimuli were generated by an Apple 7600 computer. An LCD projector (Panasonic LT562E) with a resolution of $640 \times 480$ pixels at $66 \mathrm{~Hz}$ was used to project these stimuli onto a back projection screen covering the rear open bore of the scanner. The subject viewed the stimulus on the screen using a mirror attached to the top of the head coil. As the screen was behind the subject's head, the view of the image was not impeded by the subject's body and a roughly circular area of diameter $30^{\circ}$ at the viewing distance of a

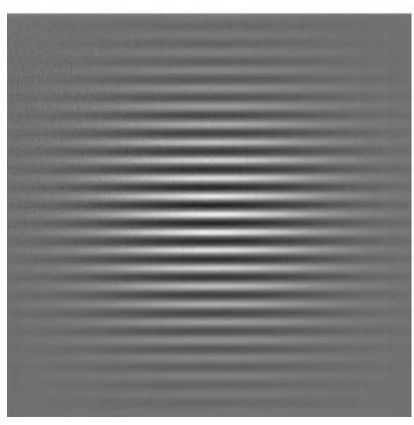

b

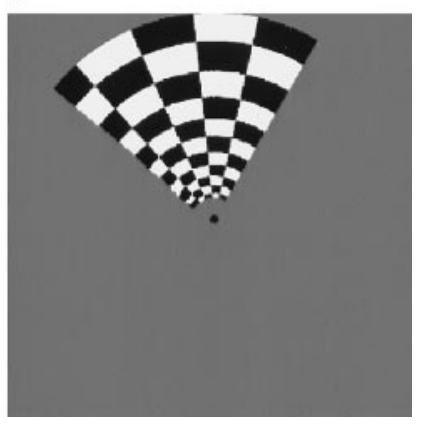

FIG. 1. Example of the images used in the study. (a) A single frame from the 2 cycles/degree sinusoid used in the tuning experiments. The image consists of a sinusoidal luminance modulation which has been multiplied by a Gaussian envelope. (b) A checkerboard wedge stimulus used for retinotopic mapping. The checks reverse polarity at $8 \mathrm{~Hz}$ to give good activation of visual cortex. The wedge rotates slowly around the central fixation at 1.1 revolutions/min.

$1.2 \mathrm{~m}$ could be seen. Visual stimulus presentation was initiated at the beginning of each $\mathrm{FMRI}$ acquisition by a TTL pulse from the computer controlling the MRI scanner.

Each subject was scanned in up to three separate sessions. Two sessions, of which some subjects completed only one and some both, were the main experimental ones, in which the stimuli designed to study the tuning functions of each visual area were presented. The third, completed by all subjects, was for the purposes of retinotopic mapping.

\section{Spatial/Temporal Frequency and Direction Sensitivity}

In the main experimental conditions, the stimuli were horizontally oriented sinusoidal luminance gratings drifting vertically upwards. Most of the experiments were performed with the grating contrast at a maximum of close to $100 \%$, but in order to study possible contrast saturation effects, some of the experiments were repeated using a low grating contrast of $5 \%$. Each grating was a single spatial frequency moving at a fixed velocity and hence contained a single temporal frequency. The grating was formed on a $544 \times 544$ pixel image matrix which subtended $12^{\circ}$ of visual angle in both height and width. In order to contain the spatial frequency spectrum to a single peak, by minimizing edge artefacts, the sinusoidal luminance profile was multiplied by a 2-D Gaussian envelope with a standard deviation of 140 pixels. A small yellow dot of diameter 10 pixels was superimposed at the center of each image, to aid visual fixation. A sample image is shown in Fig. la.

The pixel resolution and frame-rate of the projector places limits on the maximum spatial and temporal frequencies that can be presented. The maximum spatial frequency was 9 cycles/degree and the maximum 
TABLE 1

LCD Projector Response as a Function of Temporal Frequency

$\begin{array}{lcccccc}\text { Temporal frequency }(\mathrm{Hz}) & 0.0 & 1.125 & 2.25 & 4.5 & 9.0 & 18.0 \\ \text { Photodiode voltage }(\mathrm{mV}) & 195 & 195 & 195 & 185 & 180 & 180\end{array}$

temporal frequency was $18 \mathrm{~Hz}$. To assess the temporal characteristics of the projector, a photodiode was used to measure the peak to peak (i.e., black to white) intensity of the image as a function of temporal frequency. The results are shown in Table 1 and indicate that the response of the projector is reasonably flat up to $18 \mathrm{~Hz}$.

Each experimental run consisted of 54 volumeacquisitions each $3 \mathrm{~s}$ in length, giving a total run time of $162 \mathrm{~s}$. The run was divided into six blocks of $27 \mathrm{~s}$. In the first block the subject fixated the central yellow spot, which was superimposed on a uniform background of the same mean luminance as the gratings. In the second block the drifting or counterphasing grating was presented continuously for $27 \mathrm{~s}$. These two blocks were then repeated three times. This interleaved block design was designed to evoke a periodic haemodynamic response in those regions of the brain involved in processing the grating stimulus.

In one session, a variety of drifting gratings were presented. These were presented in random order but comprised two distinct sets. In the first, the spatial frequency of the grating was fixed at 2 cycles/degree, and the drift temporal frequency was set to be $0,2,5$, 9 , and $18 \mathrm{~Hz}$ in different runs. In the second, the drift temporal frequency was fixed at $5 \mathrm{~Hz}$, and the spatial frequency of the grating was set to $0.4,1,2,4$, and 7 cycles/degree in different runs.

In the other experimental session, the final tuning experiment was performed; At three different spatial frequencies (0.4, 2, 7 cycles/degree), a comparison was made between a drifting and counterphasing sinusoid with a fixed temporal frequency of $5 \mathrm{~Hz}$.

All runs were presented in a pseudo-random order for each subject, so that any arousal effects would tend to be averaged out during the data analysis process.

\section{Retinotopic Mapping Stimuli}

In the third experimental session, rotating wedge stimuli were used in order to map the boundaries between retinotopic visual areas (Sereno et al., 1995; Engel et al., 1997; Tootell et al., 1997). The checks within the wedge are of maximum (close to 100\%) contrast and flicker at $8 \mathrm{~Hz}$. The check size was scaled with eccentricity and the wedge subtended $80^{\circ}$ of visual angle. In terms of eccentricity, the image subtended $30^{\circ}$ of visual angle. An $80^{\circ}$ wedge width has been shown (Tootell et al., 1997; Smith et al., 1998) to generate good signals in all retinotopically mapped visual areas, including higher areas such as V3 and V3A. The wedge rotated in steps of $20^{\circ}$ every $3 \mathrm{~s}$ around a central fixation point. Four complete cycles were performed in each run, so each run lasted $216 \mathrm{~s}$. Four runs were carried out in each session, two with the wedge rotating anticlockwise, and two with the wedge rotating clockwise. Figure $1 b$ shows an example of the wedge stimulus used in this phase of the experiment.

\section{Anatomical I maging}

At the end of each experimental session, a wholehead anatomical scan was acquired for each subject. These were sagittal T1-weighted 3-D MP-Rage images (magnetization-prepared rapid-acquisition gradient echo; Siemens AG, Erlangen, Germany) with 1-mm cubic voxels. These anatomical volumes allowed data from the two or three sessions in each subject to be coregistered, so that data from the SF/TF tuning experiments could be combined with the drift-counterphasing datasets and the retinotopic mapping data on flattened representations of the cortical gray matter sheet (Sereno et al., 1995; Engel et al., 1997). The high resolution volume dataset also allowed functional localisations to be transformed into the Talairach bicommissural coordinate system (Talairach and Tournoux, 1988), allowing comparison with other studies.

\section{DATA ANALYSIS}

The data were analyzed and visualized using our own in-house software BrainTools (http://www. liv.ac.uk/mariarc/software.html), with three exceptions (motion correction, image registration, and cortical flattening) that are detailed below.

\section{Retinotopic Analysis}

Data were first motion corrected using imreg, part of the AF NI package (Cox, 1996) before spatial smoothing with a Gaussian filter (FWHM $=4 \mathrm{~mm}$ ) to improve signal to noise.

As the wedge rotates slowly round the visual field, each location in visual space is first stimulated and then not stimulated, in a cyclical fashion. In retinotopic visual areas, those neurons representing a specific portion of visual space are also stimulated in this fashion. 
If we assume that the haemodynamic $\mathrm{fMRI}$ response is linked to this neuronal activity, the intensity of the voxels in the fMRI scans should also show this cyclical activity.

Fourier analysis was used to ascertain the power and phase at the fundamental rotation frequency of the wedge, for each voxel in the functional dataset. The data from the four runs (two clockwise, two anticlockwise) was averaged to give the final phase values. This procedure increases the signal to noise of the resultant phase maps and, more importantly, any delay introduced by the slow hemodynamic response is averaged out by this procedure.

Finally, the phase values were converted to angles representing deviation from the upper vertical meridian in visual space. A color code was assigned to each angle, such that blue represented the upper vertical meridian, green the lower vertical meridian, and red/ orange the horizontal meridian (Engel et al., 1997).

\section{Cortical Flattening}

Although it is possible to view the calculated phase angle maps superimposed on the anatomical volume scan (See Fig. 3a for an example), the visual areas and their boundaries are folded in a complex way, which is difficult to interpret in three dimensions. However, the gray matter in the brain is in effect a folded twodimensional structure that can be unfolded computationally to reduce the visualisation problem to a two dimensional one. This is now an established procedure used by several groups (Sereno et al., 1995; Engel et al., 1997; Tootell et al., 1997; Smith et al., 1998) and we use flattening algorithms developed by Engel et al. (1997; Teo et al., 1997).

Once the "flatmap" has been constructed, the phase angles can be superimposed on it as a color overlay. Boundaries between discrete visual areas (V1, V2, V3, etc.) can be identified as regions where the direction of change of phase angle reverses. The boundaries of each visual area were identified and polygonal regions of interest (ROI) were defined which encompassed the area. These two-dimensional ROIs identify directly voxels in the 3-D anatomical scan, which are part of the visual area in question. These ROIs are then used during the analysis of the data from the tuning experiments.

In a previous study (Smith et al., 1998), we identified a possible new visual area, V3B, as a distinct region on the flatmap, identified in all subjects, and being a retinotopic area beyond the foveal end of V3. It appears to be in the same location as the kineto-occipital (KO) area described by Orban et al. (1995) as specialized for processing stimuli containing kinetically defined boundaries. However, as we cannot rule out the possibility that V3B may be the foveal portion of V3A, and as it is not yet an accepted visual area, we present results for $\mathrm{V} 3 \mathrm{~B}$ and $\mathrm{V} 3 \mathrm{~A}$ separately and also for $\mathrm{V} 3 \mathrm{~B}$ combined with $\mathrm{V} 3 \mathrm{~A}$.

\section{Tuning Data}

Data from the tuning experiments were first motioncorrected before applying spatial smoothing using a Gaussian smoothing kernel with $\mathrm{FWHM}=4 \mathrm{~mm}$. The timecourse of each voxel was then corrected for any linear trend artefact that might be present. A voxel by voxel correlation analysis, based on methods first described by Bandettini et al. (1993) and further developed by Friston et al. (1995a) was then performed. A model haemodynamic function is used as the correlating function. This model is constructed by taking a squarewave profile which follows the on-off profile of the visual stimulus, delaying it by the expected haemodynamic delay of six seconds and finally smoothing it with a Gaussian kernel with a standard deviation of three seconds. The timecourse of each voxel is smoothed with the same Gaussian, in order to improve signal to noise. This has statistical implications as it reduces the effective degrees of freedom in the correlation analysis, but we use procedures described by F riston et al. (1995a) to estimate this. Each voxel has its timecourse correlated with the model waveform. The correlation coefficient generated by this procedure can be transformed to a false probability under the null hypothesis (Cox et al., 1995) given the appropriate estimate for the effective degrees of freedom.

In this study we are interested in the magnitude of the cortical response in each visual area, as a function of the stimulus properties. The correlation coefficient and $P$ value are not appropriate measures of magnitude as they are dimensionless quantities which only describe the statistical significance of each voxel. A count of the number of voxels which achieve a particular statistical significance within a volume might be considered, but this has the disadvantage of being dependent on the spatial extent of activation, as well as the magnitude. One obvious candidate for the magnitude of the response is the variance or percentage change from the mean for each voxel. The variance so measured, however, may not be due to activity evoked by the visual stimulus, but may be due to noise or other physiological effects. In this study we use the stimulus correlated variance (SCV), which is the variance of each voxel times its correlation coefficient (Bandettini et al., 1993). If we were to formulate our analysis in terms of an equivalent General Linear Model, the SCV would be the amplitude of the main component of interest, namely that component describing the on-off stimulus profile. An alternative way of considering the $\mathrm{SCV}$ is that if the voxel timecourse and our model haemodynamic response are vectors in $n$-dimensional signal space, then the SCV represents the scalar product of these two vectors. 
However, one chooses to interpret the meaning of the $\mathrm{SCV}$, it is the natural measure of the amplitude of the evoked response. Once this quantity has been calculated for each voxel in the functional dataset, it can be represented on the gray matter flatmap for that individual. That involves the coregistration of the session where the tuning data was collected with the session in which the retinotopic data was collected. This is done by an iterative fitting procedure which translates and rotates the anatomical volume scan in one session so that it matches the similar scan collected in another session. The precise algorithm used is a crest-lines matching procedure (Subsol et al., 1998) which results in subpixel accuracy of matching. When the session-tosession transformation matrix has been calculated, it can be applied to the functional amplitude maps, so that they can be overlaid on the gray matter flatmap.

Using the previously described retinotopic mapping procedure, we have already defined ROIs on the flatmap for each visual area, so we can now calculate the average response magnitude, to each of the visual stimuli, in each of the visual areas. Note that the $30^{\circ}$ image used for retinotopic mapping was larger than the $12^{\circ}$ image used for the drifting grating stimuli. This means that we can be certain that the ROIs we have defined using retinotopic mapping will completely encompass the activity due to the drifting grating, in each visual area.

Cortical area V5/MT cannot be identified using retinotopic mapping procedures because of its relatively small size and large receptive field sizes (Tootell et al., 1995). However, V5/MT can nonetheless be identified with relative ease, because, with simple visual stimuli such as ours, it reliably appears as an island of activation in a characteristic position. Its position can be confirmed by reference to the many published locations for V5/MT in Talairach coordinates. Once the region has been identified in three-dimensional space, rather than on the flatmap, a region of interest can be defined around the area and the average response magnitude within the region can be calculated.

\section{Averaging of Data Across Subjects}

In functional imaging studies, it is usually desirable to pool functional results from several subjects. This procedure increases signal to noise and confirms that any results found are general to all. In our study, averaging across subjects has the additional advantage of controlling for attentional effects, as stimuli are presented in a pseudo-random order for each subject.

A common and powerful approach, used by the Statistical Parametric Mapping (SPM) software (F riston et al., 1995b), is to spatially normal ize the functional data to a template or model brain, which is often aligned in stereotaxic Talairach space. If all subjects are normalized or "warped" in this way, then it is a simple matter

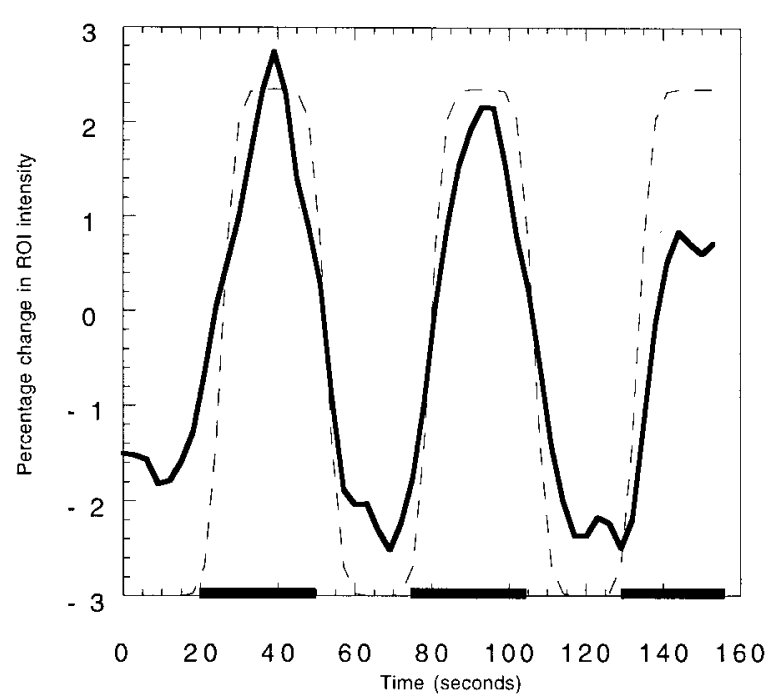

FIG. 2. Sample temporal activation waveform. This plot shows the percentage change in signal averaged over a region of interest in V1, for a single subject, as a function of time. The stimulus was a 0.4 cycles/degree sinusoid drifting at $9 \mathrm{~Hz}$. Periods during which the stimulus is present are represented by black bars. Also shown as a dashed line is the theoretical waveform used in the correlation analysis.

to combine the results of the functional analyses. However, this approach assumes that the spatial relationship between functional areas is dependent on the gross morphology of the brain itself and that by correcting for the different brain shapes of individuals we can align the functional areas of the cortex. This is clearly not the case for the human visual areas, the boundaries of which appear to depend critically on the precise sulcul geometry. For example, anatomical studies have shown that even after Talairach normalisation the position of the calcarine sulcus can vary by up to $2 \mathrm{~cm}$ (Steinmetz et al., 1990) and the size of V 1 can vary by up to a factor of 2 (Andrews et al., 1997), which is clearly much larger than the variation in brain size within the normal population.

In this study we are able to average the results of our functional analyses across subjects, within specific functional areas, because we have identified the boundaries of these areas in each individual subject using retinotopic mapping. The averaging procedure does not therefore depend on any spatial correspondence of the areas between subjects.

The tuning function in each area in each subject was first normalized so that the maximum value was 100 arbitrary units. Then the tuning functions were averaged across subjects.

\section{RESULTS}

All subjects showed stimulus-correlated haemodynamic responses in most visual areas to both drift and counterphasing gratings of $100 \%$ contrast. Figure 2 
TABLE 2

Peak Percentage Changes in Each Visual Area for Each Hemisphere in Each Subject

\begin{tabular}{|c|c|c|c|c|c|c|c|c|c|c|}
\hline Area & $\begin{array}{c}\text { Subject } \\
1 \text { left }\end{array}$ & $\begin{array}{l}\text { Subject } \\
1 \text { right }\end{array}$ & $\begin{array}{c}\text { Subject } \\
2 \text { left }\end{array}$ & $\begin{array}{l}\text { Subject } \\
2 \text { right }\end{array}$ & $\begin{array}{c}\text { Subject } \\
3 \text { left }\end{array}$ & $\begin{array}{l}\text { Subject } \\
3 \text { right }\end{array}$ & $\begin{array}{c}\text { Subject } \\
4 \text { left }\end{array}$ & $\begin{array}{l}\text { Subject } \\
4 \text { right }\end{array}$ & $\begin{array}{c}\text { Subject } \\
5 \text { left }\end{array}$ & $\begin{array}{l}\text { Subject } \\
5 \text { right }\end{array}$ \\
\hline V1 & $3.6 \%$ & $2.3 \%$ & $1.4 \%$ & $2.0 \%$ & $3.3 \%$ & $3.6 \%$ & $2.5 \%$ & $2.4 \%$ & $3.3 \%$ & $2.3 \%$ \\
\hline $\mathrm{V} 2 \mathrm{~d}$ & $1.6 \%$ & $1.5 \%$ & $1.5 \%$ & $1.7 \%$ & $2.3 \%$ & $2.2 \%$ & $1.9 \%$ & $2.6 \%$ & $2.4 \%$ & $1.8 \%$ \\
\hline V2v & $2.4 \%$ & $1.7 \%$ & $1.6 \%$ & $1.8 \%$ & $3.0 \%$ & $2.1 \%$ & $2.1 \%$ & $1.7 \%$ & $2.9 \%$ & $2.8 \%$ \\
\hline V3 & $2.2 \%$ & $2.0 \%$ & $2.0 \%$ & $2.0 \%$ & $2.1 \%$ & $1.9 \%$ & $2.1 \%$ & $2.3 \%$ & $2.5 \%$ & $2.3 \%$ \\
\hline V3A & $2.1 \%$ & $2.2 \%$ & $1.9 \%$ & 0 & $2.2 \%$ & $3.0 \%$ & $2.2 \%$ & $2.2 \%$ & $2.8 \%$ & $1.5 \%$ \\
\hline V3B & $3.1 \%$ & $2.0 \%$ & $2.4 \%$ & 0 & $1.7 \%$ & $1.9 \%$ & $1.6 \%$ & $2.1 \%$ & $0.5 \%$ & 1.4 \\
\hline V4 & $1.5 \%$ & 0 & $1.9 \%$ & $2.0 \%$ & $1.5 \%$ & 0 & $1.2 \%$ & 0 & $2.3 \%$ & $2.0 \%$ \\
\hline V5 & $1.8 \%$ & $1.4 \%$ & $0.8 \%$ & $1.3 \%$ & $2.8 \%$ & $2.7 \%$ & $1.6 \%$ & $1.0 \%$ & $1.1 \%$ & $2.0 \%$ \\
\hline VP & $2.1 \%$ & $0.9 \%$ & $1.4 \%$ & $1.8 \%$ & $2.3 \%$ & $1.6 \%$ & $2.0 \%$ & $1.0 \%$ & $3.0 \%$ & $2.7 \%$ \\
\hline
\end{tabular}

shows a typical functional response from $\mathrm{V} 1$ in one of our subjects. At $5 \%$ contrast, only responses in area V5/MT could be reliably identified. Table 2 shows the maximum percentage change elicited in each visual area, for each of the subjects used in the spatiotemporal tuning experiments.

Figure 3 shows retinotopic and tuning data for one of our subjects. In Fig. 3a we can see the phase data superimposed on the 3-D anatomical volume. In Fig. 3b the same data are shown represented on the flattened left occipital cortex. The borders between the adjacent visual areas can be clearly visualized as stripes of uniform color, curving away from the foveal representation, which is marked with a "*." In Fig. 3c, we show the data from one of the tuning experiments, overlaid on the same flatmap.

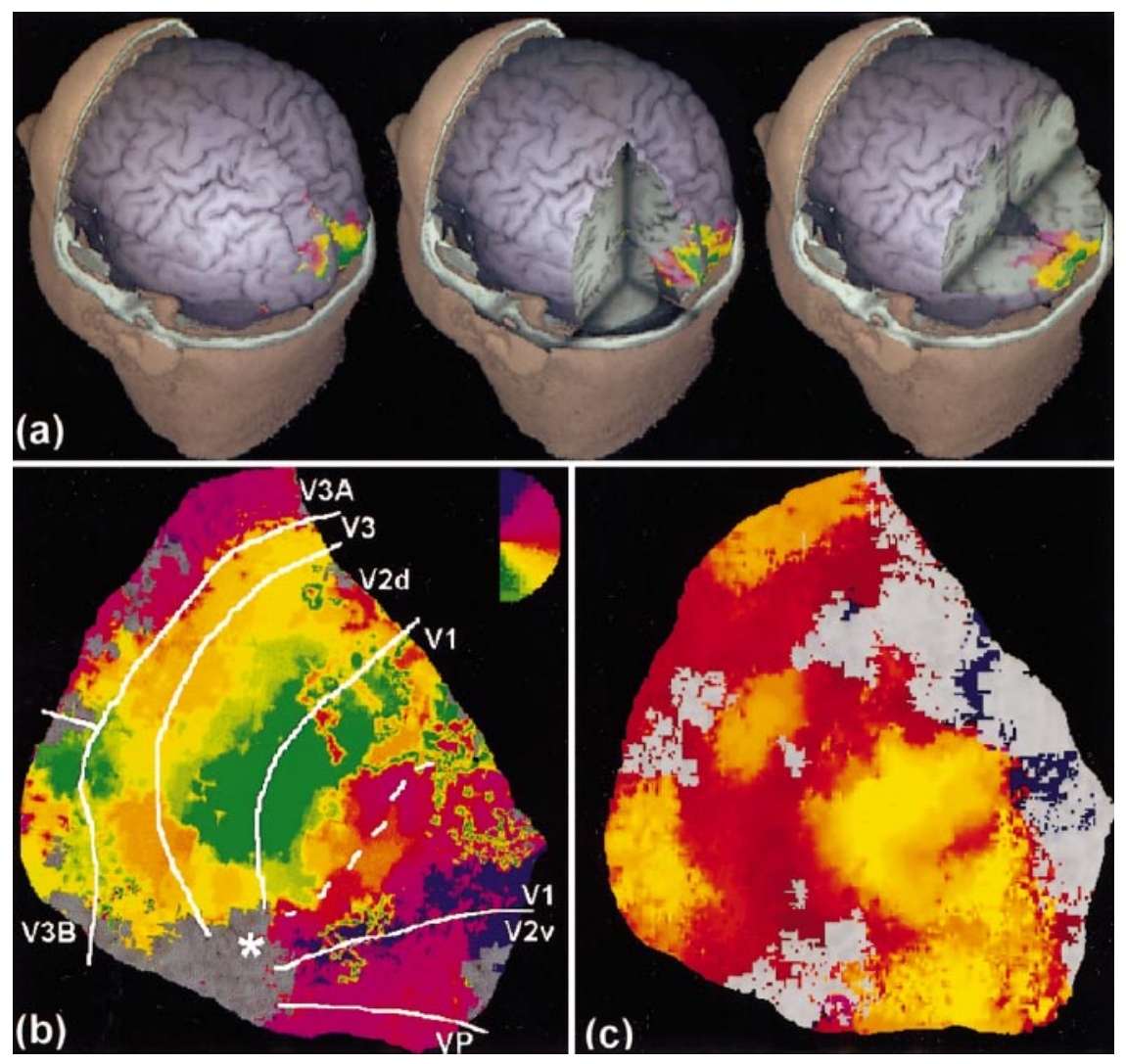

FIG. 3. This figure shows an example of how retinotopic data and flatmaps were used in calculating the tuning functions. (a) The retinotopic data for a single subject as a color overlay on several 3-D views of the anatomical volume. The boundaries between visual areas are difficult to ascertain. (b) The same data as a color overlay on the flattened left occipital lobe of this subject. The col or key in the top right shows the relationship between the pseudo-col or representation and visual angle. The white '*' marks the foveal representation. In (c) we see the functional data from one of the tuning experiments $(0.4$ cycles/degree, $9 \mathrm{~Hz}$ ), overlaid on the same flatmap. The boundaries shown on (b) can be used to calculate the magnitude of the haemodynamic response in each visual area, for the data shown in (c). 

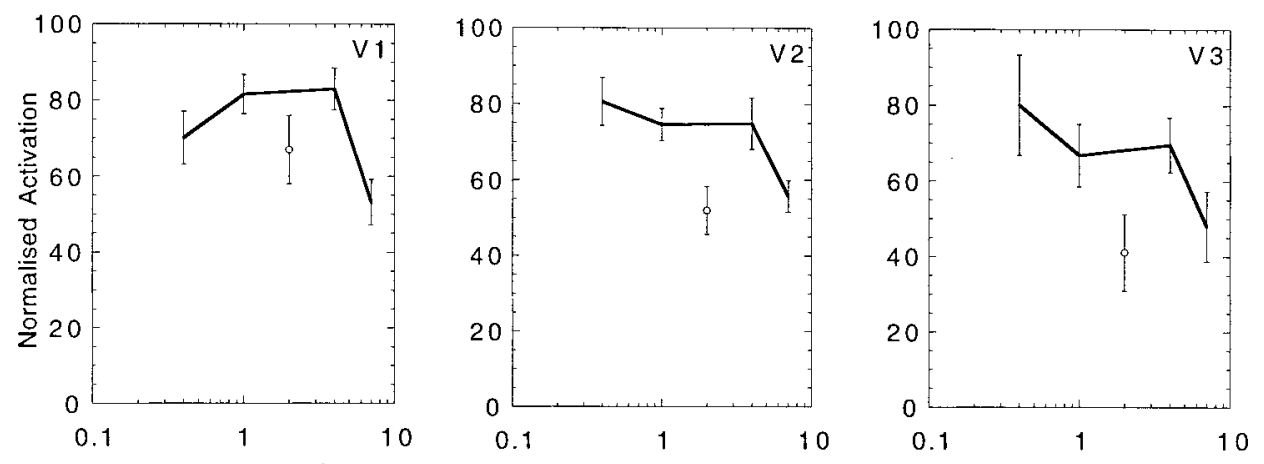

Spatial frequency (cycles/degree)
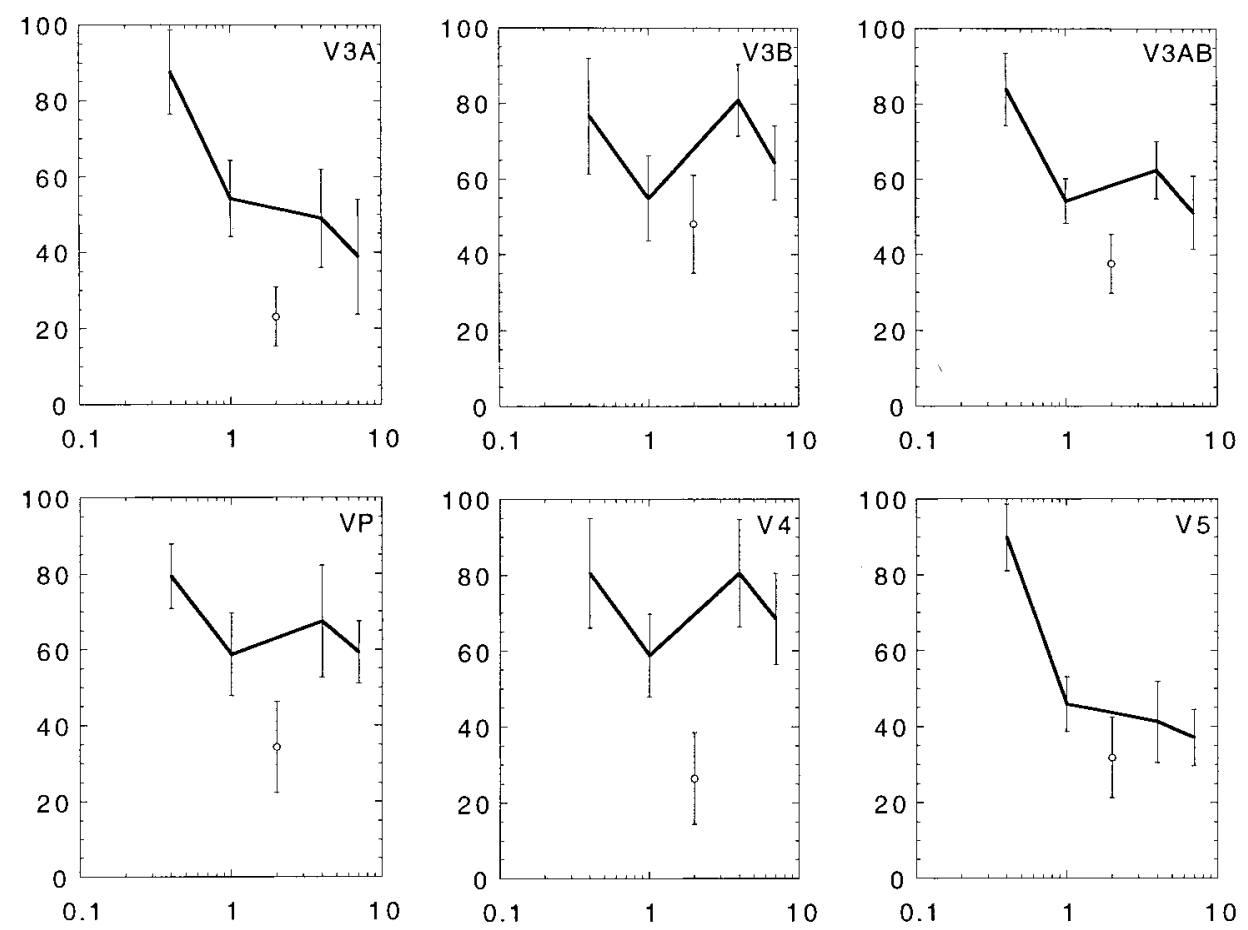

FIG. 4. Normalized fMRI activation levels in each visual area, averaged over 10 hemispheres in 5 subjects, elicited by a 5 Hz drifting sinusoid of various spatial frequencies. The error bars represent the standard error on each point. The 2 cycles/degree point is shown as an open symbol because adaptation from the temporal frequency experiment may have reduced sensitivity at this point.

\section{Spatial Frequency Tuning}

Figure 4 shows the spatial frequency tuning functions for each of the visual areas we identified. All of the visual areas show a noticeable dip in the tuning function at 2 cycles/degree. The most likely explanation for this dip is that all the temporal frequency tuning experiments are carried out at 2 cycles/degree, so it is possible that some adaptation has taken place, resulting in a reduced response at 2 cycles/degree. For this reason, the 2 cycles/degree data-point is shown as an open circle on the figures, rather than being included in the tuning curve.

In $\mathrm{V} 1$, the curve rises slowly to peak at between 1 and 4 cycles/degree before dropping rapidly at 7 cycles/ degree. Foster et al. (1985) showed that cells in macaque V1 were optimally tuned at between 2 and 4 cycles/degree and that the cell response fell rapidly above 4 cycles/degree. The mean optimal response was 2.2 cycles/degree. The general form of our tuning curve is very similar to Foster's data. MEG experiments by Fylan (personal communication) show the MEG response in V1 to drifting luminance gratings is also band-pass with a peak at 4-6 cycles/degree.

V2, V3, and VP appear more low-pass tuned for spatial frequency. Foster et al. (1985) found that the neuronal population in macaque V2 showed an increased preference for lower spatial frequencies compared to the cells in V1, with a mean optimal value of 0.65 cycles/degree. Levitt et al. (1994) found that the mean optimal spatial frequency for macaque V2 cells was 1.4 cycles/degree. Although these studies agree that a substantial proportion of $\mathrm{V} 2$ cells prefer low 
spatial frequencies, they disagree on whether V2 is low-pass tuned for spatial frequency or band-pass tuned. As we have no fMRI tuning data below 0.4 cycles/degree we cannot contribute to this debate, however over the range we tested (0.4 to 7 cycles/degree) our tuning functions are in general agreement with both studies. Our peak spatial frequency for V2 (0.4 cycles/degree) is closer to that of Foster et al. than Levitt et al.

V3A shows a strong preference for low spatial frequencies, with the peak response at 0.4 cycles/degree. In macaque $\mathrm{V} 3 \mathrm{~A}$, most cells exhibit low-pass tuning with a mean peak of 1.75 cycles/degree (Gaska et al., 1988). In macaque $V 3$, neurons show a preference for lower spatial frequencies than V2 cells (Gegenfurtner et al., 1997) with a mean peak response at 0.4 cycles/ degree, and almost no cells responding above 4 cycles/ degree. This suggests that in macaque, V3 cells are tuned for lower spatial frequencies than V3A. In our fMRI tuning data, V3A appears to be more strongly tuned for low spatial frequencies than V3. So our data support the hypothesis that $V 3$ and V3A might be reversed in comparison with macaque. Functional imaging of V3A in humans (Tootell et al., 1997) has also revealed that it has large receptive field sizes, which would correspond to our result showing a preference in V3A for low spatial frequencies.

The $\mathrm{fMRI}$ response in V3B is more flat across the tested spatial frequencies. The fact that the spatial frequency curves for V3A and V3B are dissimilar might, at first sight, lead us to the conclusion that they are indeed separate visual areas. However, if V3B was the foveal portion of V3A we would expect the cells in this region to be more sensitive to higher spatial frequencies. The results from this experiment do not, therefore, provide evidence for or against the hypothesis that V3B is a separate visual area. For this reason, we have included tuning curves for $V 3 A, V 3 B$ and an averaged curve for V3AB. In V3AB, the spatial frequency tuning curve is still low-pass, but with a more constant response at higher spatial frequencies than the curve for V3A alone.

The spatial frequency tuning curve for V4 is essentially flat across all the tested spatial frequencies. It is interesting to note that the curve is very similar to that obtained for V3B (see above). It is also interesting to note in passing that on the flatmap, $V 3 B$ represents only the lower visual field, while V4 represents the upper visual field. So far, no dorsal equivalent for V4 has been identified in humans (Tootell et al., 1997).

As expected the response in V5/MT is low-pass tuned for spatial frequency, and drops rapidly as the spatial frequency increases above 0.4 cycles/degree. This is consistent with the reported predominance of magnocellular (M) cells within V5/MT which are known to be preferentially sensitive to low spatial frequency targets. Anderson et al. (1996) used MEG to investigate a

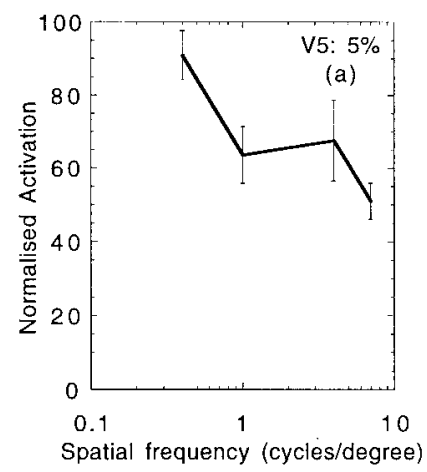

b

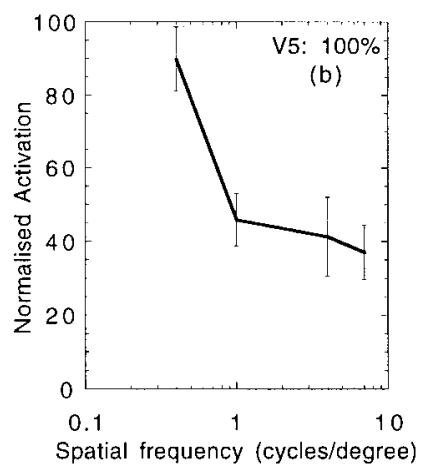

FIG. 5. (a) Normalized fMRI activation levels in V5/MT, elicited by a drifting sinusoid of contrast $5 \%$, as a function of spatial fre quency (at $5 \mathrm{~Hz}$ ). The curve is the average of 9 hemispheres in 5 subjects. The error bars represent the standard error on the mean values. In (b) we reproduce the tuning curve at $100 \%$ contrast, taken from Fig. 4, for the purposes of comparison.

spatial frequency tuning in V5/MT and found the area was strongly tuned for low spatial frequencies, with no measurable response at 4 cycles/degree. Our results are similar, but we were able to measure weaker haemodynamic responses at even the highest spatial frequency we tested (7 cycles/degree).

Figure 5 a shows the spatial frequency tuning functions for $\mathrm{V} 5 / \mathrm{MT}$, at $5 \%$ grating contrast. The results show that the spatial frequency tuning function is not dependent on the contrast we have chosen. Although physiological data lead us to expect that response saturation will occur at $100 \%$ contrast, at least in V5, our data suggest that if it does occur the saturation level appears to vary with spatial frequency, in proportion to sensitivity at low contrast.

We can compare the fMRI spatial frequency tuning curves with psychophysical studies. Kelly (1979) measured the spatiotemporal threshold surface using a system stabilised for eye movements. In Fig. 9a we show a model psychophysical contrast sensitivity function for a grating drifting at $5 \mathrm{~Hz}$. We have used the model of Kelly (1979, his Eq. (8)), which he derived from psychophysical data. At this temporal frequency, the curve is bandpass with a faster falloff at higher spatial frequencies than for low spatial frequencies. This is similar to our $\mathrm{fMRI}$ response amplitude curves in V1. The Kelly model predicts a peak at $1.8 \mathrm{cycles} /$ degree for this temporal frequency.

\section{Temporal Frequency Tuning}

Figure 6 shows the temporal frequency tuning functions for each visual area. All areas show essentially the same pattern, and appear broadly band-pass tuned with a peak at approximately $9 \mathrm{~Hz}$. Again, there is a pronounced dip in the data at $5 \mathrm{~Hz}$, which happens to be the temporal frequency used in the spatial fre- 

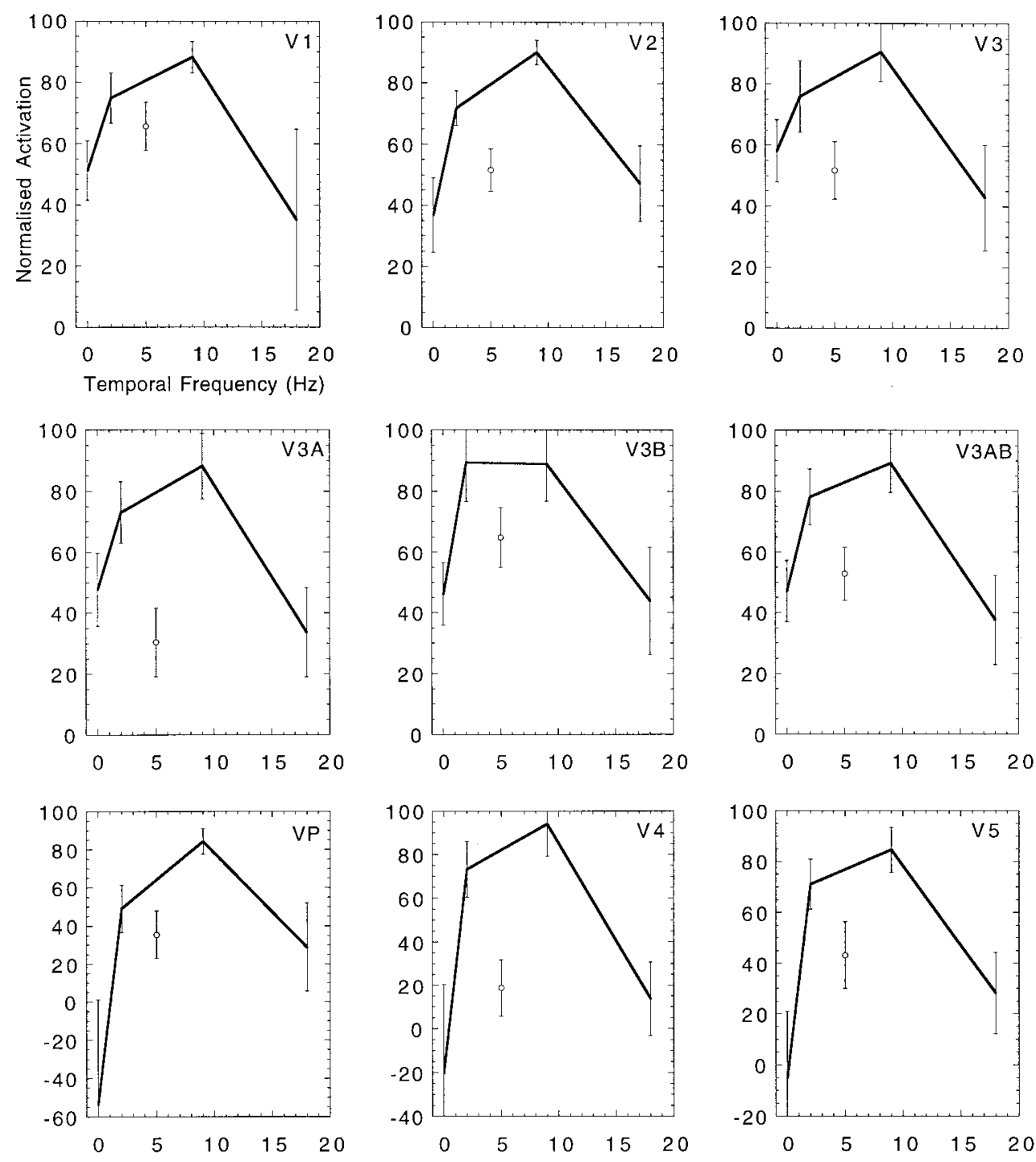

FIG. 6. Normalized fMRI activation levels in each visual area, averaged over 10 hemispheres in 5 subjects, elicited by a 2 cycles/degree drifting sinusoid of various temporal frequencies. The error bars represent the standard error on each point. The $5 \mathrm{~Hz}$ point is shown as an open symbol because adaptation from the spatial frequency tuning experiment may have reduced sensitivity at this point.

quency tuning phase of the experiment. As with the spatial frequency data, it is possible that adaptation has caused a reduced response at $5 \mathrm{~Hz}$, and for this reason the $5 \mathrm{~Hz}$ data is shown as an open circle symbol, rather than being included in the tuning curve.

All areas show an increase in response amplitude between a stationary grating and one moving at the optimal frequency of $9 \mathrm{~Hz}$. In V1, V2, V3, and V3A, the increase is a factor of approximately 1.5-2. In VP, V4, and V5/MT we were not able to measure any significant response amplitude from a stationary grating.

It is interesting to compare our tuning curves with the limited number of single cell studies in macaque that have used drifting gratings. Foster et al. (1985) showed that most neurons in $\mathrm{V} 1$ and $\mathrm{V} 2$ responded to temporal frequencies up to around $8 \mathrm{~Hz}$ with a sharp decrease in the number of cells responding above $8 \mathrm{~Hz}$. Similarly in V2, Gegenfurtner et al. (1996, 1997) showed that the majority of cells in V2 responded over the range $1-10 \mathrm{~Hz}$ (with a median value of $4.2 \mathrm{~Hz}$ ) and that at higher temporal frequencies the number of responding cells fell rapidly. Levitt et al. (1994) found that most V2 cells were sensitive to drift rates of between 3 and $4 \mathrm{~Hz}$. In V3, Gegenfurtner et al. (1997) found a median value for the peak sensitivity of cells at $6 \mathrm{~Hz}$. Again, many cells responded between 1 and 10 $\mathrm{Hz}$ before the number of responding cells fell rapidly. These studies in macaque all found that the tuning curves were very similar for V1, V2, and V3 cells and the form of the tuning curve is similar to the one we have found. However, the studies show that the peak of the optimum temporal frequency histogram for these 
cells is at approximately 3- $6 \mathrm{~Hz}$, not the $9 \mathrm{~Hz}$ we have found.

In terms of other functional imaging studies, Kwong et al. (1992), using fMRI, and Fox et al. (1985), using PET, have previously shown that the response in V1 to a flicker stimulus is band-pass tuned for temporal frequency, with a peak response at $8 \mathrm{~Hz}$. The curves they obtained were extremely similar to the ones we present here. In these two studies, the spatial properties of the stimuli used was completely different to the single sinusoid we use in this study, yet the temporal frequency tuning curves they obtained are essentially the same.

Using MEG, Anderson et al. (1996) found that the magnitude of the current generator in V5/MT was band-pass tuned for the temporal frequency of a drifting sinusoid, with the curve appearing approximately flat between 1 and $30 \mathrm{~Hz}$. In one subject, the maximum response was at $8 \mathrm{~Hz}$. Also using MEG, Fylan et al. (1997) found band-pass tuned responses in V1, with a peak response at 8-10 Hz. Morrone et al. (1996) measured the amplitude of visual evoked electrical potentials (VEPs) using a drifting grating stimulus. They found that in adult subjects, the peak response occurred when the grating temporal frequency was 10 $\mathrm{Hz}$.

Again we can compare our temporal frequency tuning data in V1 with psychophysical data. Kelly (1979) showed that at 2 cycles/degree the contrast detection curve is band-pass tuned with temporal frequency and peaks at between 3 and $5 \mathrm{~Hz}$. This model curve is shown in Fig. 9b. This detection curve is similar to our tuning curve in V1, however, Kelly's model predicts a peak sensitivity at $3.2 \mathrm{~Hz}$ compared to our $9 \mathrm{~Hz}$ peak. Similarly, Watson and Turano (1995) found that the optimal motion stimulus was at $5 \mathrm{~Hz}$.

However, the precise position of the peak sensitivity increases with luminance of the stimulus (Kelly 1961). For the relatively high luminance used in this study, and for velocities above $1 \%$ s the optimal temporal frequency was found by Burr and Ross (1982) to be approximately $10 \mathrm{~Hz}$, which is close to our maximum value.

\section{Direction Sensitivity}

Several electrophysiological studies (for example Albright, 1984; Albright et al., 1984) have indicated that cells in the area V5/MT are characterised by their preference for stimulus motion in a particular direction. We tested whether fMRI responses can be used to characterise the amount of direction sensitivity in each visual area. This can be best shown by calculating the ratios between responses to counterphase flickering gratings and those drifting in a single direction. If the responses are similar (i.e., ratios approximately equal to 1 ) the area responds equally well to flicker and motion, whereas if the ratio is significantly greater than 1 then the area prefers unidirectional motion. Figure 7 shows that in all visual areas, gratings drifting in a single direction produce at least as much activity as gratings that are counterphasing (flickering) at the same temporal frequency. In many cases, they produce greater activation. The ratios we obtained are quite modest, between $(0.9 \pm 0.2)$ and $(1.8 \pm 0.3)$ with the highest ratio occurring in V5/MT at the highest spatial frequency. Heeger et al. (1999) also compared drifting and counterphasing gratings in $\mathrm{V} 1$ and V5/MT at 0.4 cycles/degree (their Fig. 3). In V1 their ratios were very close to 1.0 for both subjects studied. In V5/MT, the average ratio for the two subjects was approximately 1.7, compared to our value at this spatial frequency of (1.2 \pm 0.2$)$.

This increased activation in our study appears to be dependent on spatial frequency, and varies across areas. Visual areas can be placed in two categories: (1) V1, V2, V3, and V3AB, which show the largest increase in activity at low spatial frequencies (2) VP, V4, and V5, which have the largest ratios at the highest spatial frequency of 7 cycles/degree. The error measures on these ratios are often large so it is difficult to be confident that these differences are real, rather than a result of sampling error. However, F oster et al. (1985) assessed the directional sensitivity of single neurons in macaque V1 and V2 and found that the incidence of cells preferring motion in a particular direction was highest in neurons that preferred lower spatial frequencies.

Figure 8a shows a graph of the same drift versus counterphasing ratio in V5/MT, but this time the contrast was $5 \%$ rather than $100 \%$. Again, this demonstrates that the ratio behaves in the same way at two different image contrasts.

\section{DISCUSSION}

By systematic variation of the properties of a visual stimulus and coregistration with the functional anatomy of each individual subject, ascertained using retinotopic mapping, we have demonstrated that the hemodynamic response magnitude varies as a function of spatial/temporal frequency and the amount of directed motion. Our results are in good agreement with previous functional imaging studies of V1 and V5/MT.

Our results for both the spatial and temporal frequency tuning curves are in good accord with those of electrophysiological studies that use similar stimuli, i.e., drifting gratings. However, these studies predict a lower peak for the temporal frequency tuning curve (3- $6 \mathrm{~Hz}$ ) than the $8-10 \mathrm{~Hz}$ we have found. Although we can be confident of the spatial characteristics of our stimulus, we cannot be certain that low temporal frequency artefacts, arising from the temporal properties of the LCD elements, are not introduced by the projec- 

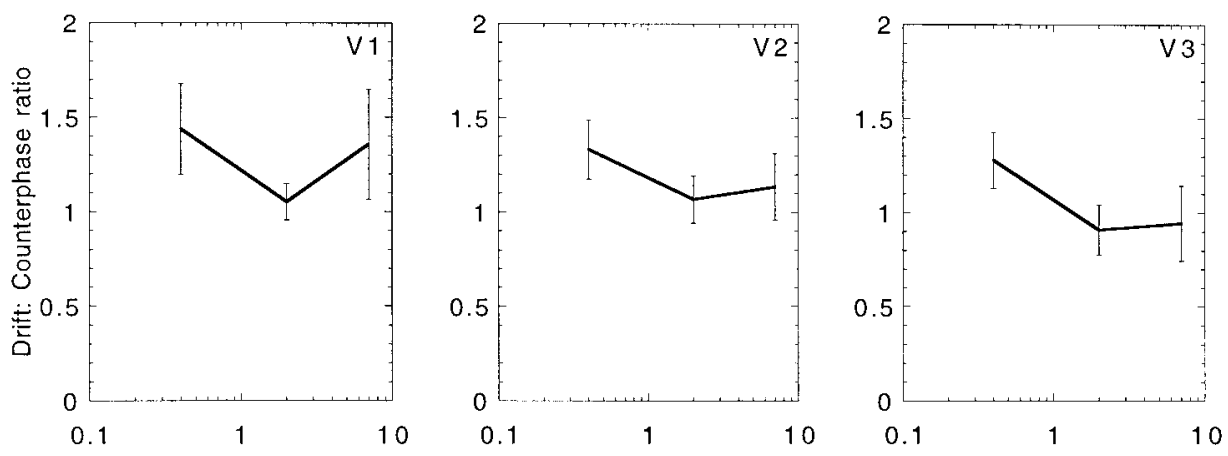

Spatial frequency (cycles/degree)
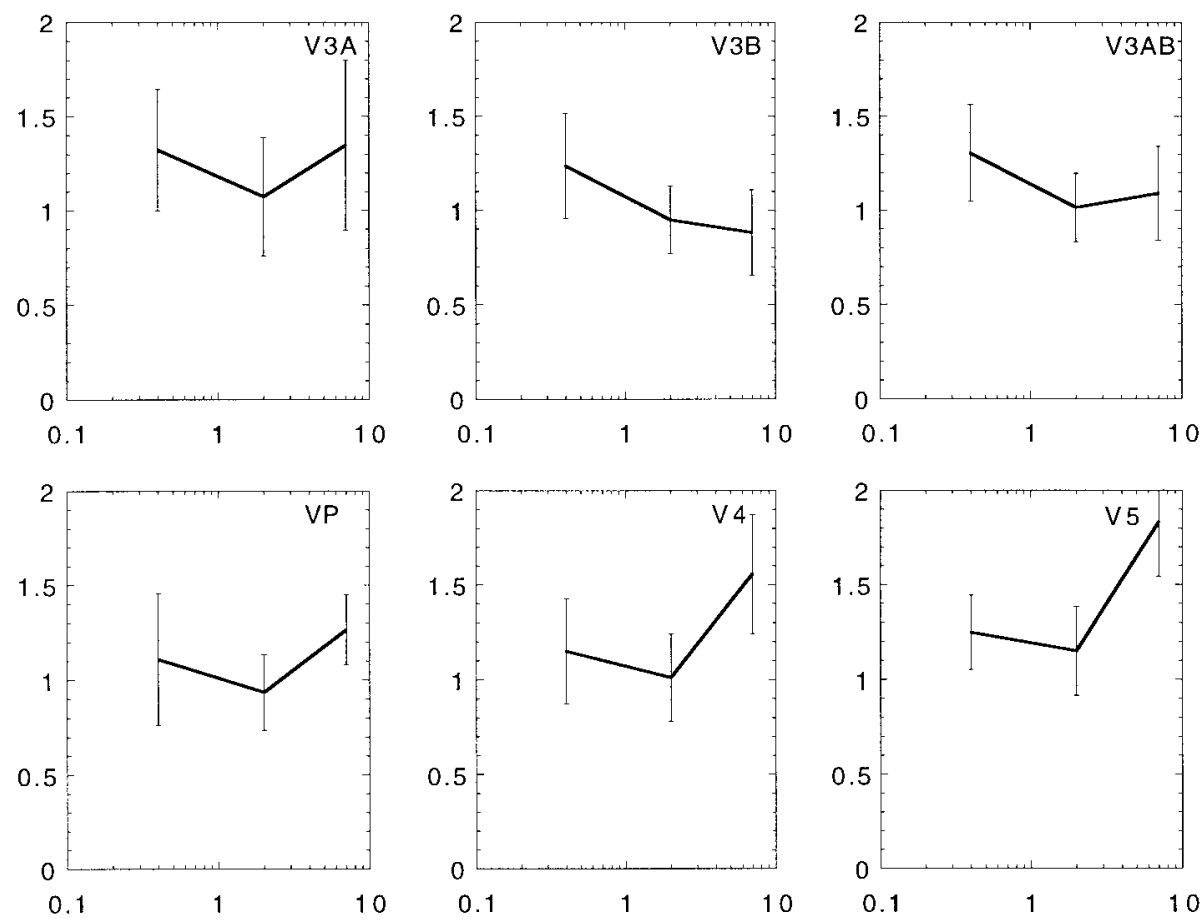

FIG. 7. Graphs showing the Drift versus Counterphase ratio in each visual area, as a function of spatial frequency, for 10 hemispheres in 5 subjects. The sinusoid either drifted or counterphased at a temporal frequency of $5 \mathrm{~Hz}$. The error bars represent the standard error on the ratio.

tor. However, the 8-10 Hz peak has been previously found in other functional imaging studies using different presentation modalities and also in numerous psychophysical studies.

It appears that in terms of neuron receptive fields, spatial and temporal frequency are separable quantities, i.e., for a particular neuron, the optimal spatial frequency is independent, over a large range, of the temporal frequency used to stimulate it (Foster et al., 1985). This suggests that parametric variation of spatial and temporal frequency is the best way to investigate the response of neurons in each visual area. Despite this, many single-cell studies vary the speed of an object such as a bar, across the receptive field of the cell. This has the undesirable result that the optimum velocity depends on the spatial frequency content of the object used. Such studies (Felleman and Van Essen, 1987; Cheng et al., 1994; Maunsell and Van Essen, 1983; Rodman and Albright, 1987) find that different visual areas have different velocity tuning curves, and the peak velocities are at significantly higher velocities than our $4.5 \%$ s. Similar results have been found in fMRI studies of velocity tuning in V3A and V5/MT (Chawla et al., 1998, 1999). These findings are in conflict with our data, but the difference is almost certainly due to the use of a spatially broadband stimulus, rather than the single spatial frequency used in this study. For example, it is known that more cells in V5/MT show a preference for lower spatial frequencies compared to VI. If those cells had the same temporal frequency tuning characteristic as V 1 cells (as our data seems to show), but were detecting only the low spatial 
a

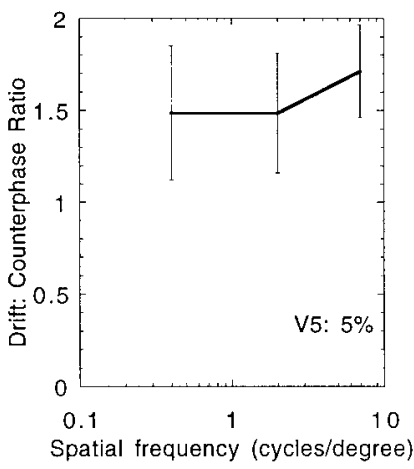

b

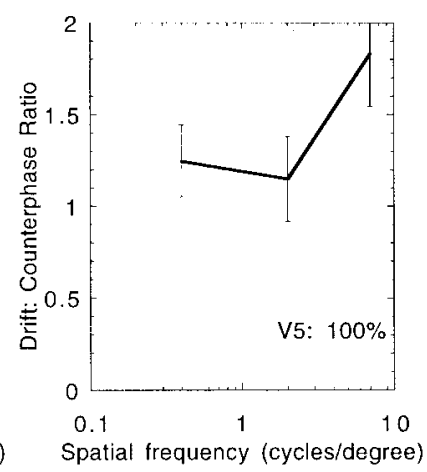

FIG. 8. (a) Graph showing the Drift versus Counterphase ratio in V5/MT as a function of spatial frequency. The sinusoid was of contrast $5 \%$ and either drifted or counterphased at a temporal frequency of $5 \mathrm{~Hz}$. The curve is the average of 8 hemispheres from 4 subjects. The error bars represent the standard error on the ratio. In (b) we have reproduced the figure for $100 \%$ contrast, taken from Fig. 7, for the purposes of comparison.

frequency components in the broadband bar stimulus, then the V5/MT cells would appear to be tuned for a higher velocity than V1 cells.

A remarkable result is that the temporal frequency tuning curves, in each visual area, appear more or less identical. One possible explanation is that neuronal projections between each visual area have resulted in us measuring an overall tuning function. The fact that we find different spatial frequency tuning functions in different visual areas would seem to militate against this. A second possibility is that the temporal fre-

quency function we are measuring is nothing to do with the intrinsic neuronal populations, but is a generic feature of the haemodynamics. This is unlikely as the characteristic haemodynamic time constants are of the order of 3- $6 \mathrm{~s}$, rather than the $9 \mathrm{~Hz}$ optimum we are measuring. Also, MEG and VEP studies show that the macroscopic evoked electrical response, which involves no hemodynamics, varies in the same way. Finally, it is interesting to note that one of the predominant rhythms of visual cortex is the Alpha rhythm at 8-10 $\mathrm{Hz}$. If this rhythm represented an intrinsic resonant frequency for the human visual cortex, then it might be that driving the system with a stimulus containing temporal frequencies at this optimal frequency would result in the maximum response.

In visual areas V1, V2, and V5/MT, our spatial frequency tuning curves correspond well with what might be predicted from electrophysiological studies in macaque. However, the story is more complicated in V3 and V3A. Recently, evidence has emerged that V 3 and V3A in macaque may not truly correspond to their human counterparts (Tootell et al., 1997; Smith et al., 1998), and our data for V3 and V3A support the hypothesis that $\mathrm{V} 3$ and $V 3 \mathrm{~A}$ might be reversed in human compared to macaque. Also, our spatial frequency tuning curves for V3A are extremely similar to those for V5/MT which is known to be part of the magnocellular pathway. This is interesting in that it has been postulated that macaque V3 might be considered as part of the parietal motion analysis pathway (Gegenfurtner et al., 1997) and receives a significant input from layer $4 \beta$ a

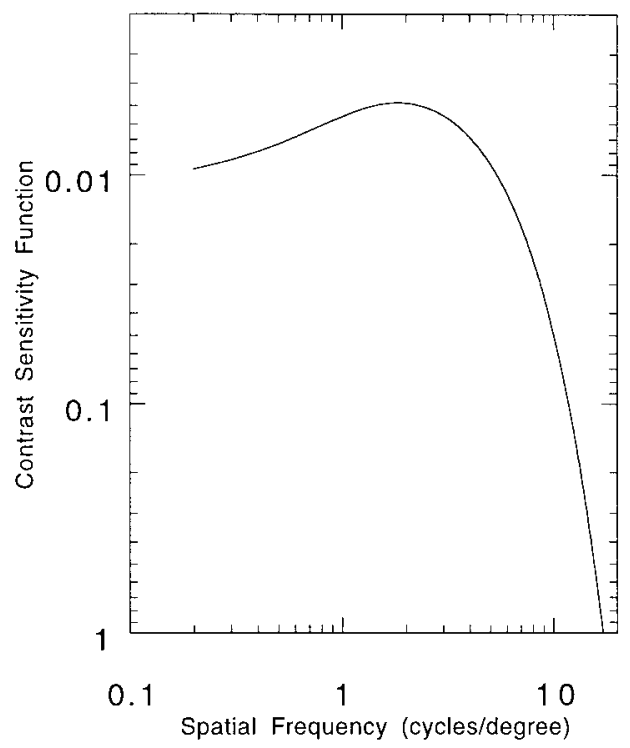

b

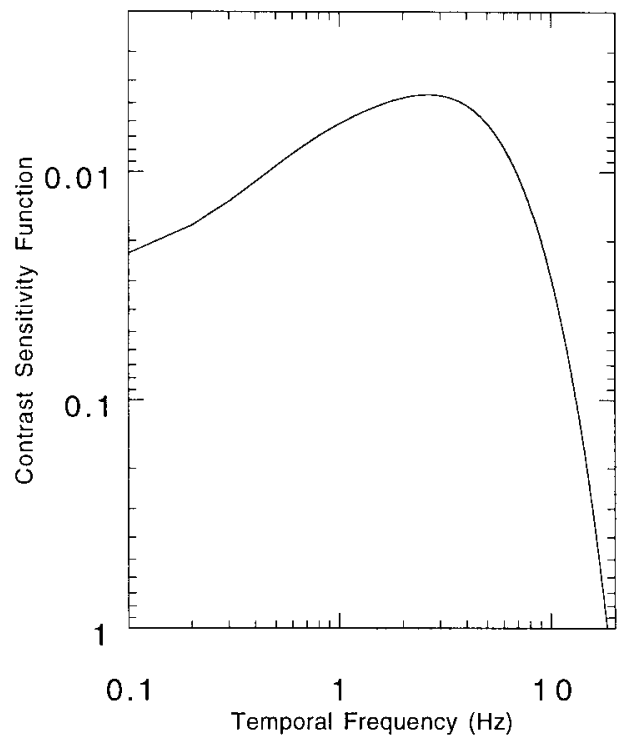

FIG. 9. This figure shows the contrast sensitivity functions for detection of a drifting grating, plotted as a function of spatial frequency (a) and temporal frequency (b). In (a) the temporal frequency of the drifting grating is $5 \mathrm{~Hz}$, while in (b) the spatial frequency of the drifting grating is 2 cycles/degree. The parameters of the psychophysical model were fitted from psychophysical contrast sensitivity data by Kelly (1979). 
of $\mathrm{V} 1$, which is dominated by magnocellular input. In addition, histological evidence shows many similarities between macaque $\mathrm{V} 3$ and $V 5 / M T$. If V3A serves the same role in human, then we might expect that V3A would show similar tuning properties to V5/MT and this is indeed what we find. At present this must be considered speculative as the histological evidence linking V3A and V5/MT in humans does not yet exist (Tootell et al., 1997).

A limitation of our study is that we have used high contrast stimuli, in order to elicit a response with a high signal to noise ratio. Psychophysical studies of motion detection thresholds necessarily use much lower image contrasts. Despite this difference, we have found an excellent correspondence between psychophysical measures of spatial-temporal frequency sensitivity and the haemodynamic tuning curves in V1. The good correspondence between our fMRI response magnitudes and psychophysical measures support the previously reported findings of Boynton et al. (1999) that $\mathrm{fMRI}$ responses reflect the response of a large neuronal population and that it is therefore the pooled activity of a large number of neurons which is responsible for the detection performance of the human visual system.

The purpose of comparing responses to drifting and counterphasing gratings was to assess the motion specificity of each visual area. Moving visual stimuli have been used in numerous fMRI studies and they generally produce strong activation. Tootell et al. (1997) attempted to quantify motion specificity by comparing responses to moving and stationary versions of the same stimulus. They found moving/stationary response ratios close to unity in V1 and V2, but much higher in V3A and, in particular, V5.

However, neurophysiological studies in primate show that many neurons respond well to temporal modulation but are not very sensitive to direction. So part of the fMRI response may arise from temporal luminance modulation at point locations in the image, rather than from movement.

In a previous fMRI study (Smith et al., 1998) of second-order motion, we obtained similar results to Tootell when comparing responses to moving and static images, but obtained much lower ratios (less motion specificity) when responses to motion superimposed on dynamic noise were compared with dynamic noise alone. In this case the ratio never exceeded 2:1 even in V5. This suggests that motion-specific activity does indeed constitute only part of the activation related to the temporal structure of the stimulus.

Arguably, the best test of motion specificity is the drifting versus counterphase comparison used both in this study and a recent study by Heeger et al. (1999). This is a strong test, since the only difference between the stimuli is that the temporal energy has a single direction in one case and is directionless (equal in opposite directions) in the other. In both studies, the ratios obtained never exceeded 2:1, even in V5, and in many cases were not significantly different from 1:1. The results we present here and previous studies both therefore suggest that motion sensitivity, particularly in V5 but also in other areas, is grossly overestimated by moving versus static comparisons.

Flickering stimuli such as counterphasing gratings can be considered mathematically as comprising balanced motion components in opposite directions. Counterphase gratings might therefore stimulate more direction-sensitive neurons than drifting ones, because two groups of neurons with opposite preferences will be activated instead of one. However, computational models (e.g., Adelson and Bergen, 1985) generally assume that motion signals in opposite directions at the same location are subtracted at the detection stage to give a single, net motion signal. If this motion opponency occurs, then direction-sensitive neurons should be unresponsive to counterphase gratings. Physiological data, such as they are, suggest that the truth is between the two. Qian and Andersen (1995) illustrate direction-selective neurons in primate $V 1$ that respond less well to counterphase gratings than to gratings drifting in the preferred direction, but much better than to drift in the null (antipreferred) direction. Thus, comparing counterphase and drift responses, although better than comparing moving and static responses, may not give a clean indication of motion specificity. Quantifying motion sensitivity with $\mathrm{FMRI}$ is not straightforward and rests on the assumptions one chooses to make. In particular, drawing conclusions about the proportion of direction-sensitive neurons in a given visual area must be done with great care. However, our data and the study of Heeger et al . (1999) both seem to support the hypothesis that motion opponency and direction selectivity play a role in the processing of visual motion in human cortex.

\section{ACKNOWLEDG MENTS}

This work was supported by grants from The Wellcome Trust to A.T.S. and from Deutsche Forschungsgemeinschaft (Grant GR98815) to M.W.G. We are grateful to Professor J. Hennig for use of the MRI scanning facilities at the Radiologische Universitätskliniken, Freiburg. M.W.G. was supported by the Schilling Foundation. We thank Dr. B. Wandell for providing support in the use of cortical flattening software. We also thank Dr. R. M. Rutschmann for measurements of the LCD projector temporal frequency characteristics.

\section{REFERENCES}

Adelson, E. H., and Bergen, J . R. 1985. Spatiotemporal energy models for the perception of motion. J . Opt. Sci. Am. A. 2: 284-299.

Albright, T. D. 1984. Direction and orientation selectivity of neurons in visual area MT of the macaque. J . Neurophysiol. 52: 1106-1130.

Albright, T. D., Desimone, R., and Gross, C. G. 1984. Columnar organization of directionally selective cells in visual area MT of the macaque. J . Neurophysiol. 51: 16-31. 
Allman, J. M., and Kaas, J . H. 1971. A representation of the visual field in the caudal third of the middle temporal gyrus of the owl monkey (Aotus trivirgatus). Brain Res. 31: 85-105.

Anderson, S. J ., Holliday, I. E., Singh, K. D., and Harding, G. F. A. 1996. Localization and functional analysis of human cortical area V5 using magneto-encephalography. Proc. R. Soc. Lond. Biol. 263: 423- 431.

Andrews, T. J ., Halpern, S. D., and Purves, D. 1997. Correlated size variations in human visual cortex, lateral geniculate nucleus, and optic tract. J . Neurosci. 17(8): 2859-2868.

Bandettini, P. A., J esmanowicz, A., Wong, E. C., and Hyde, J. S. 1993. Processing strategies for time-course data sets in functional MRI of the human brain. Magn. Reson. Med. 30: 161-173.

Boynton, G. M., Demb, J . B., Glover, G. H., and Heeger, D. J . 1999. Neuronal basis of contrast discrimination. Vision Res. 39: 257269.

Britten, K. H., Shadlen, M. N., Newsome, W. T., and Movshon, J . A. 1992. The analysis of visual motion: A comparison of neuronal and psychophysical performance. J . Neurosci. 12(12): 4745- 4765.

Burr, D. C., and Ross, J . 1982. Contrast sensitivity at high velocities. Vision Res. 23: 3567-3569.

Campbell, F. W., and Robson, J. G. 1968. Application of Fourier analysis to the visibility of gratings. J . Physiol. 197: 551-566.

Chawla, D., Phillips, J ., Buechel, C., Edwards, R., and Friston, K. J . 1998. Speed-dependent motion-sensitive responses in V5: An fMRI study. Neurol mage 7(2): 86-96.

Chawla, D., Buechel, C., Edwards, R., Howseman, A., J osephs, O., Ashburner, J., and Friston, K. J. 1999. Speed-Dependent Responses in V5: A Replication Study. Neuroimage 9(5): 508-515.

Cheng, K., Hasegawa, T., Saleem, K. S., and Tanaka, K. 1994. Comparison of neuronal selectivity for stimulus speed, length, and contrast in the pre-striate visual cortical areas V4 and MT of the macaque monkey. J . Neurophysiol. 71(6): 2269-2280.

Cox, R. W., J esmanowicz, A., and Hyde, J . S. 1995. Real-time functional magnetic resonance imaging. Magn. Reson. Med. 33(2): 230-236.

Cox, R. W. 1996. AFNI: Software for analysis and visualization of functional magnetic neuroimages. Comput. Biomed. Res. 29: 162173.

Dubner, R., and Zeki, S. M. 1971. Response properties and receptive fields of cells in an anatomically defined region of the superior temporal sulcus in the monkey. Brain Res. 35: 528-532.

Engel, S. A., Rumelhart, D. E., Wandell, B. A., Lee, A. T., Glover, G. H., Chichilnisky, E.J ., and Shadlen, M. N. 1994. fMRI of human visual cortex. Nature 369: 525.

Engel, S. A., Glover, G. H., and Wandell, B. A. 1997. Retinotopic organization in human visual cortex and the spatial precision of functional MRI. Cereb. Cortex 7: 181-192.

Felleman, D. J ., and Van Essen, D. C. 1987. Receptive field properties of neurons in area V3 of macaque monkey extrastriate cortex. J. Neurophysiol. 57(4): 889-920.

Foster, K. H., Gaska, J. P., Nagler, M., and Pollen, D. A. 1985. Spatial and temporal frequency selectivity of neurones in visual cortical areas V1 and V2 of the macaque monkey. J. Physiol. (Lond.) 365: 331-363.

Fox, P. T., and Raichle, M. E. 1985. Stimulus rate determines re gional blood flow in striate cortex. Ann. Neuro. 17: 303-305.

Fox, P. T., Miezin, F. M., Allman, J. M., Van Essen, D. C., and Raichle, M. E. 1987. Retinotopic organization of human visual cortex mapped with positron-emission tomography. J . Neurosci. 7(3): 913-922.

Friston, K. J ., Holmes, A. P., Poline, J . B., Grasby, P. J ., Williams, S. C. R., Frackowiak, R. S. J ., and Turner, R. 1995a. Analysis of fMRI time series revisited. Neuroimage 2: 45-53.
Friston, K. J ., Ashburner, J ., Poline, J . B., Frith, C. D., Heather, J. D., and Frackowiak, R. S. J . 1995b. Spatial Registration and Normalization of Images. Hum. Brain Mapp. 2: 165-189.

Fylan, F., Holliday, I. E., Singh, K. D., Anderson, S. J ., and Harding, G. F. A. 1997. Magnetoencephalographic investigations of human cortical area V1 using color stimuli. Neuroimage 6: 47-57.

Gaska, J. P., J acobson, L. D., and Pollen, D. A. 1988. Spatial and temporal frequency selectivity of neurons in visual cortical area V3A of the macaque monkey. Vision Res. 28(11): 1179-1101.

Gegenfurtner, K. R., Kiper, D. C., and Levitt, J . B. 1997. Functional properties of neurons in macaque area V3. J . Neurophysiol. 77(4): $1906-1923$.

Heeger, D. J ., Boynton, G. M., Demb, J . B., Seidemann, E., and Newsome, W. T. 1999. Motion opponency in visual cortex. J . Neurosci. 19(16): 7162-7174.

Kelly, D. H. 1961. Visual Responses to time dependent stimuli. 1. Amplitude sensitivity measurements. J . Opt. Soc. Am. 51: 422- 429.

Kelly, D. H. 1979. Motion and Vision. II. Stabilized spatio-temporal threshold surface. J . Opt. Soc. Am. 69(10): 1340-1349.

Kulikowski, J . J ., and Tolhurst, D. J . 1973. Psychophysical evidence for sustained and transient mechanisms in human vision. J . Physiol. 232: 149-163.

Kwong, K. K., Belliveau, J. W., Chesler, D. A., Goldberg, I. E., Weisskoff, R. M., Poncelet, B. P., Kennedy, D. N., Hoppel, B. E., Cohen, M. S., and Turner, R. 1992. Dynamic magnetic resonance imaging of human brain activity during primary sensory stimulation. Proc. Natl. Acad. Sci. USA 89: 5675-5679.

Levitt, J . B., Kiper, D. C., and Movshon, J . A. 1994. Receptive fields and functional architecture of macaque V2. J . Neurophysiol . 71(6): 2517-2542.

Maunsell, J . H., and Van Essen, D. C. 1983. Functional properties of neurons in middle temporal visual area of the macaque monkey. 1. Selectivity for stimulus direction, speed and orientation. J . Neurophysiol. 49(5): 1127-1147.

Maunsell, J . H. R., Nealey, T. A., and DePriest, D. D. 1990. Magnocellular and parvocellular contributions to responses in the middle temporal visual area (MT) of the macaque monkey. J . Neurosci. 10: 3323-3334.

Morrone, M. C., Fiorentini, A., and Burr, D. C. 1996. Development of the temporal properties of visual evoked potentials to luminance and colour contrast in infants. Vision Res. 36(19): 3141-3155.

Orban, G. A., Dupont, P., De Bruyn, B., Vogels, R., Vandenberghe, R., and Mortelmans, L. 1995. A motion area in human visual cortex. Proc. Natl. Acad. Sci. USA 92: 993-997.

Qian, N., and Andersen, R. A. 1995. V1 responses to transparent and non-transparent motions. Exp. Brain Res. 103: 41-50.

Rodman, H. R., and Albright, T. D. 1987. Coding of visual stimulus velocity in area MT of the macaque. Vision Res. 27(12): 2035-2048.

Sereno, M. I., Dale, A. M., Reppas, J. B., Kwong, K. K., Belliveau, J. W., Brady, T. J ., Rosen, B. R., and Tootell, R. B. H. 1995. Borders of multiple visual areas in humans revealed by functional magnetic resonance imaging. Science 268: 889-893.

Shadlen, M. N., Britten, K. H., Newsome, W. T., and Movshon, J . A. 1996. A computational analysis of the relationship between neuronal and behavioral responses to visual motion. J. Neurosci. 16(4): 1486-1510.

Smith, A. T., Greenlee, M., Singh, K. D., Kraemer, F. M., and Hennig, J . 1998. The processing of first- and second-order motion in human visual cortex assessed by functional magnetic resonance imaging (fMRI). J . Neurosci. 18(10): 3816-3830.

Steinmetz, H., Furst, G., and Freund, H-J . 1990. Variation of perisylvian and calcarine landmarks within stereotaxic proportional coordinates. AJ NR 11: 1123-1130.

Subsol, G., Thirion, J. P., and Ayache, N. 1998. As scheme for automatically building three-dimensional morphometric anatomi- 
cal atlases: application to a skull atlas. Med. Image Anal. 2(1): 37- 60.

Talairach, J ., and Tournoux, P. 1988. Co-Planar Stereotaxic Axis of the Human Brain. Thieme, New York.

Teo, P. C., Sapiro, G., and Wandell, B. A. 1997. Creating connected representations of cortical gray matter for functional MRI visualization. IEEE Trans. Med. Imaging 16(6): 852-863.

Tootell, R. B. H., and Taylor, J . B. 1995. Anatomical evidence for MT and additional cortical visual areas in humans. Cereb. Cortex. 1: 39-55.

Tootell, R. B. H., Reppas, J . B., Kwong, K. K., Malach, R., Born, R. T., Brady, T. J ., Rosen, B. R., and Belliveau, J. W. 1995. Functional analysis of human MT and related visual cortical areas using magnetic resonance imaging. J . Neurosci. 15: 3215-3230.

Tootell, R. B. H., Mendola, J . D., Hadjikhani, N. K., Ledden, P. J ., Liu, A. K., Reppas, J. B., Sereno, M. I., and Dale, A. M. 1997.
Functional analysis of $\mathrm{V} 3 \mathrm{~A}$ and related areas in human visual cortex. J . Neurosci. 17: 7060-7078.

Watson, J. D. G., Myers, R., Frackowiak, R. S. J ., Hajinal, J . V., Woods, R. P., Mazziotta, J . C., Shipp, S., and Zeki, S. 1993. Area V5 of the human brain: Evidence from a combined study using positron emission tomography and magnetic resonance imaging. Cereb. Cortex 3: 79-94.

Watson, A. B., and Turano, K. 1995. The optimal motion stimulus. Vision Res. 35(3): 325-336.

Zeki, S. M. 1974. Functional organization of a visual area in the posterior bank of the superior temporal sulcus of the rhesus monkey. J . Physiol. (Lond.) 236: 549-573.

Zeki, S., Watson, J . D. G., Lueck, C. J ., Friston, K. J ., Kennard, C., and Frackowiak, R. S. J. 1991. A direct demonstration of functional specialization in the human visual cortex. J . Neurosci. 11: 641- 649. 